\title{
Resistencia a la fractura de postes de flbra de vidrio vs postes colados en dientes anteriores. Revisión sistemática
}

\author{
Fracture Resistance of fiberglass post vs cast post in anterior \\ teeth. Systematic review
}

Mónica Ruiz-Matorel ${ }^{1 凶}$, Marco Fidel Pardo-Betancourt ${ }^{2 凶}$, Gustavo Jaimes-Monroy ${ }^{3 凶} \underline{\text { CvLAC }}$, Elibeth Muñoz-Martínez ${ }^{4 凶}$, José Edwin Palma-Medina ${ }^{4} \bowtie$

1. Rehabilitadora Oral. Docente del área clínica. Universidad Antonio Nariño. Grupo de investigación en salud oral. Ciudad, País?

2. Endodoncista. Docente del área clínica. Universidad Antonio Nariño. Grupo de investigación en salud oral. Ciudad, País?

3. Odontólogo. Lic. en Biología. Especialista en Epidemiología, Magister en Bioquímica. Docente investigador. Universidad Antonio Nariño. Grupo

de investigación en salud oral. Ciudad, País?

4. Estudiantes décimo semestre. Universidad Autónoma de México. México.

Fecha correspondencia:

Recibido: noviembre de 2015.

Aceptado: junio de 2016.

\section{Forma de citar:}

Ruiz-Matorel M, Pardo-Betancourt

MF, Jaimes-Monroy G, Muñoz-

Martínez E, Palma-Medina JE.

Resistencia a la fractura de postes de fibra de vidrio vs postes colados en dientes anteriores. Revisión sistemática de la literatura. Rev. CES Odont 2016; 29(1): 45-56.

\section{Open Acces}

(C) Derecho de autor

Licencia creative commons

Ética de publicaciones

Revisión por pares Gestión por Open Journal System

ISSN 0120-971X e-ISSN 2215-9185

Comparte

\section{Resumen}

Los dientes anteriores con tratamiento endodóntico y con pérdida de estructura dental usualmente son restaurados con postes de fibra de vidrio o con postes colados, y son susceptibles a presentar fallas que pueden llegar a ocasionar la pérdida dental por no ser rehabilitables. Se propone aquí, identificar cuál entre los dos tipos de postes: prefabricados de fibra de vidrio o postes colados, es más resistente a la fractura debido a las fuerzas biomecánicas compresivas o tensionales que se suceden en los dientes anteriores rehabilitados con coronas respecto a la fractura del poste, de la raíz o el desalojo de este. Se realizó una revisión sistemática de la literatura, con información recolectada desde las bases de datos de los buscadores: PubMed, Scielo, Lilacs y Science Direct. Noventa y tres artículos sobre el tema fueron tamizados según los criterios de selección. Cuatro cumplieron con este procedimiento y se encontró que la resistencia a la fractura tiende a ser mayor en dientes restaurados con postes colados, pero cuando se presenta una fractura es desfavorable para el retratamiento restaurativo. En el caso de los postes de fibra de vidrio se presenta mejor resistencia a la fractura y presentan un patrón por lo general más favorable para un retratamiento en caso de éste fracaso.

Palabras clave: Resistencia a la fractura, Poste colado, Poste pre-fabricado en fibra de vidrio, Diente endodónticamente tratado.

\section{Abstract}

Anterior teeth with endodontic treatment and loss of tooth structure are usually restored with glass fiber posts or cast cores, which are susceptible to present failures that may potentially cause tooth loss because they are not able to be restored again. It is proposed here to identify which of the two types of posts: prefabricated fiberglass or cast posts, is more resistant to fracture due to biomechanical forces of compression that occur in the anterior teeth restored with crowns respect to fracture post, root or evic- 
tion of this. A systematic review of the literature was conducted with information gathered from the databases of search engines: PubMed, Scielo, Lilacs and Science Direct. Ninety three articles on the topic were screened according to the selection criteria. Four cumplied with this procedure and found that fracture resistance tends to be grater in teeth restored with cast posts, but when a tracture accors is untaorubale for a restorative retreat. In the case of fiberglass posts and greater remaining height structure better fracture resistance is presented, and they present a pattern usually more favorable for retreatment in case of fracture.

Keywords: Resistance fracture, Post cast, Prefabricated glass fiber post, Endodontically treated teeth.

\section{Introducción}

Los retenedores intraradiculares tipo postes, son empleados para restaurar dientes tratados endodónticamente desde 1870, su función primaria es soportar y conectar la restauración coronal con el remanente radicular y distribuir las fuerzas (1-4). Los postes colados conforman una unidad que es elaborada mediante una impresión del conducto radicular previamente preparado en su parte interna quedando una porción de gutapercha intacta para el adecuado selle apical; se confeccionan mediante un poste en cera o acrílico de autocurado que luego será colado con una aleación

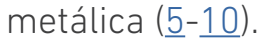

Los retenedores intraradiculares deben ser estéticamente compatibles con la corona y los tejidos circundantes, en casos de dientes anteriores muy destruidos, los postes colados pueden generar un tono gris metálico tanto en la estructura dental remanente como en el tejido gingival $(11,12)$. Mediante el empleo de postes en fibra de vidrio es posible la rehabilitación con coronas más translúcidas (13,14). Los postes colados pueden afectar el resultado estético de una corona completamente cerámica, debido a ello han ido ganando popularidad los postes de fibra de vidrio por sus propiedades estéticas y biomecánicas favorables $(\underline{15}, 16)$, los cuales han sido diseñados para ser cementados mediante técnicas adhesivas dentro del conducto radicular y el remanente dental para que posteriormente sea reconstruido con una resina compuesta (17-20).

A través de los años, muchos sistemas de postes prefabricados han sido introducidos en el mercado y empleados por los profesionales alrededor del mundo, con la ventaja que disminuyen el tiempo de labor clínica, el número de procedimientos, el trabajo del laboratorio y reducen los costos; como desventaja a este sistema está la falta de evidencia de su durabilidad a largo plazo al ser comparado con los postes colados tradicionales (21-23). Las cargas que recibirán los dientes dependen de la ubicación de ellos en la cavidad oral. Los dientes anteriores son sometidos a cargas horizontales u oblicuas y los dientes posteriores son sometidos a cargas verticales $(\underline{24}, \underline{25})$. Por esta razón para los dientes anteriores es muy importante que el poste posea un módulo de elasticidad similar a la dentina $(26,27)$. Una fractura se considera un fracaso común en los dientes tratados endodónticamente debido a las cargas oclusales. Cuando se emplea postes metálicos es posible provocar estas fracturas radiculares, con más frecuencia que cuando se utilizan postes de fibra de vidrio con un módulo de elasticidad similar al de la dentina (28). Según Chuang y col. en el 2012 reportan la microfiltración marginal como uno de los indicadores que se manifiesta previamente a la pérdida de retención y que puede ser en ocasiones, un factor que se asocia con la fractura radicular (29-31). 
En consecuencia, el objetivo de este estudio fue realizar una revisión sistemática de la literatura con el fin de identificar cuál entre los dos tipos de retenedores intrarradiculares postes colados o postes prefabricados de fibra de vidrio es más resistente a las fuerzas biomecánicas en dientes anteriores rehabilitados con coronas respecto a la fractura del retenedor, de la raíz o el desalojo de este.

\section{Estrategia de búsqueda}

Se realizó una revisión sistemática de la literatura para discutir de manera detallada sobre la conveniencia del uso de postes colados metálicos o de fibra de vidrio en dientes anteriores, según la evidencia hallada. Para esto se tamizó la información en cuatro bases de datos de la literatura: PubMed, Scielo, Lilacs y Science Direct producidos desde los años 2005 hasta el 2015.

Para la recolección de los datos se utilizaron las palabras clave de búsqueda en español: resistencia a la fractura, poste colado, poste en fibra de vidrio y diente endodónticamente tratado, y sus respectivas traducciones al inglés: Fracture resistance, Cast post, glass fiber post, endodontically treated teeth.

Para la selección de los estudios, se incluyeron los artículos que cumplieran con los criterios de inclusión: escritos en inglés y español, de estudios experimentales in vitro que evaluaran la resistencia a la fractura de postes frente a cargas oclusales en dientes anteriores tratados con endodoncia, publicados desde el año 2005 a 2015. Se seleccionaron los escritos que presentaron muestras mayores de $(n=10)$ dientes. Como criterios de exclusión: estudios realizados en modelos animales, estudios no experimentales e in vivo y estudios sin resultados confirmados con criterios estadísticos suficientes.

Los artículos seleccionados en esta etapa fueron evaluados de manera independiente, frente a los criterios de inclusión y exclusión en texto completo por tres revisores de las áreas de endodoncia, rehabilitación y epidemiología. Posteriormente, se registraron los resultados en una escala de construcción propia basada en la escala PEDro (32) que resultó ser la más cercana al objetivo del estudio, con 11 ítems individuales puntuados con 1 o 0 según cumpliera o no con el criterio revisado. Se seleccionaron aquellos artículos con puntajes mayores a 10. Los artículos que fueron excluidos, con puntajes cercanos, recibieron discusión grupal de los revisores para decidir su selección o no.

Existe una producción importante de artículos en esta temática en las bases de datos revisadas. La búsqueda de información inicial obtuvo fundamentos para el análisis de revistas de diversa naturaleza, entre las más relevantes encontró 44 artículos publicados en la revista de prostodoncia dental Journal of Prosthetic Dent, actualmente en el cuartil 2 de la clasificación Scimago Journal and Country Rank (SJR); 15 artículos en el Journal of Dentistry clasificada en el cuartil 1 según SJR; 12 en la revista Dental Material en cuartil 1 en la clasificación SJR, y 8 en International Endodontic Journal clasificada en el cuartil 1 en SJR, 5 artículos en el Journal of Oral Rehabilitation; estos como los principales aportes, y en menor proporción con menos de 5 artículos otras revistas de carácter internacional.

De los 93 artículos hallados, se excluyeron 64 por no ser estudios in vitro y 23 más se descartaron por ser estudios en dientes diferentes a los anteriores; por tamaño de muestra insuficiente fueron descartados 2 artículos del estudio. Al final del tamizaje quedaron 4 artículos cómo insumo de la revisión sistemática de la literatura (Figura 1). 


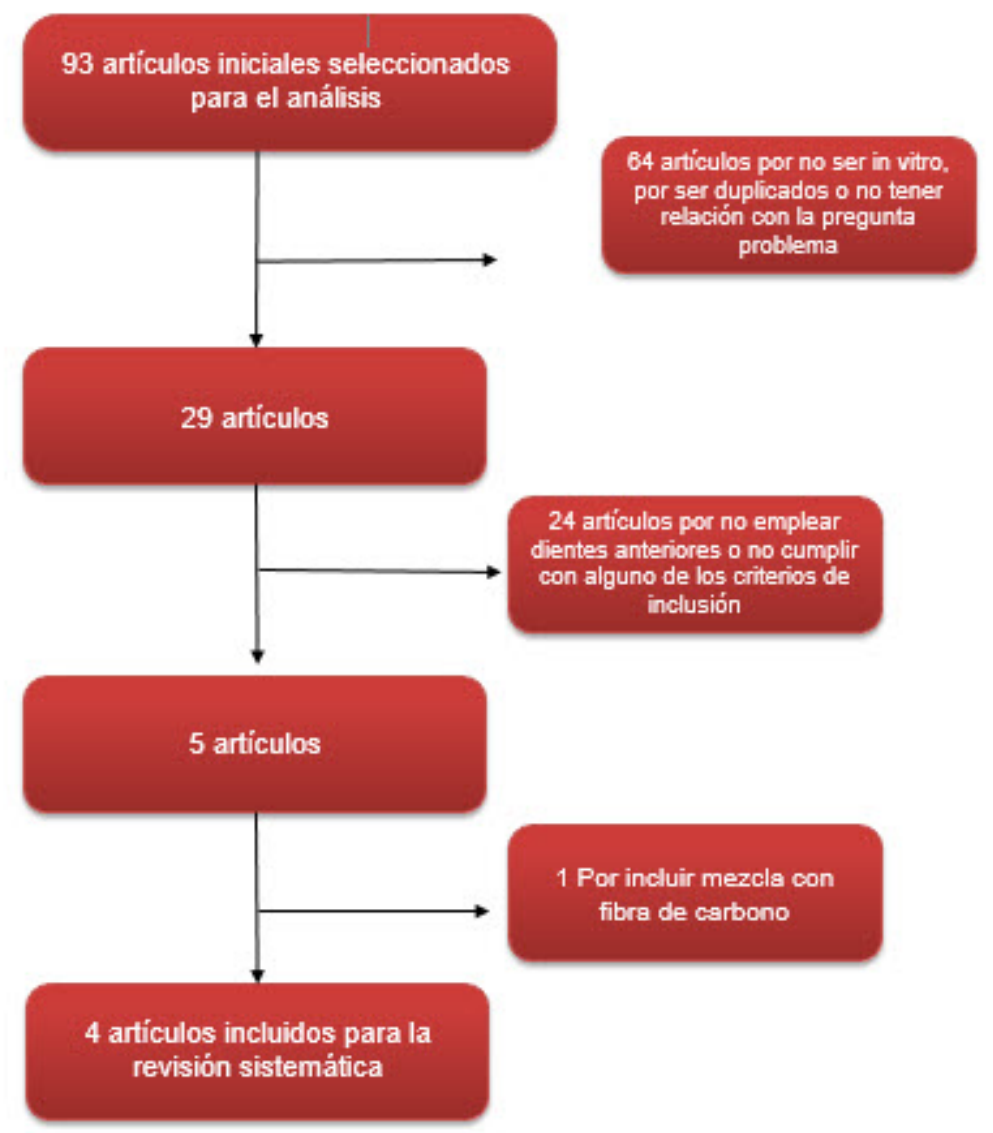

Figura 1. Diagrama de flujo del proceso de selección de los artículos para la revisión sistemática de la literatura.

La metodología de los estudios tenía en común la aplicación de fuerzas controladas sobre los modelos radiculares con los postes, y adicionalmente, coincidían en la respuesta esperada: fractura o desalojo del poste, en los dientes anteriores. El objetivo final de estos artículos, se centró en el reconocimiento de las mejores condiciones de respuesta, que indicaran el mejor pronóstico del diente para su posterior rehabilitación.

\section{Hallazgos desde los artículos tamizados}

Maroulakos y col. en el 2015 (34) realizaron un estudio en 30 dientes anteriores superiores tratados endodonticamente que fueron divididos en 3 grupos, el primero con postes colados en oro; el segundo con postes prefabricados de titanio y muñón con resina compuesta y el tercero con postes prefabricados de fibra de vidrio reforzado con cuarzo y muñón en resina compuesta. Las muestras se trataron en termociclador. Se verificó el modelo de fractura por imágenes de micro tomografía computarizada y se sometieron a fallas mediante una máquina universal de pruebas. Los datos fueron analizados con ANOVA de una sola vía, en donde se observa la respuesta sobre la variable resistencia a la fractura ocasionada por el tipo de material. Todos los grupos mostraron diferencias en la prueba de ANOVA $p=0,002$. Los postes colados en oro mostraron más alta resistencia a la fractura, comparado con los demás con una media de $174 \mathrm{~N}$; el modo principal de fracaso para los postes colados en oro y para los postes prefabricados con titanio y muñón con resina compuesta, fue la fractura radicular, mientras que para los postes de fibra de vidrio reforzado con cuarzo y muñón con resina compuesta, fue el desalojo del poste. 
Chuang y col. en el 2010 (33), estudiaron en una muestra de 60 incisivos, la longitud adecuada para la retención intrarradicular del poste y adicionalmente las fuerzas y tensiones soportadas en el interior de la raíz en referencia a su respuesta de manera comparativa con la longitud, entre postes de fibra de vidrio, de carbono y prefabricado de acero inoxidable. Los dientes extraídos fueron tratados con endodoncia, restaurados y divididos en 3 grupos; el primer grupo con postes prefabricados de acero inoxidable, el segundo grupo con postes prefabricados de fibra de carbono y el tercer grupo con postes prefabricados de fibra de vidrio. Luego se subdividieron en dos grupos para tratar las condiciones de longitud de preparación radicular a 5 y $10 \mathrm{~mm}(n=10)$. Los dientes fueron sometidos a condiciones de termociclado y luego se aplicaron cargas oblicuas al inicio de $100 \mathrm{~N}$ fuerza sobre la superficie lingual de la corona a $2 \mathrm{~mm}$ del borde incisal y a $135^{\circ}$ de angulación. Para el análisis estadístico se realizó ANOVA de 2 vías, $(p=0.05)$ para comparar tipo de material y longitud del poste a 5 y $10 \mathrm{~mm}$ sobre la variable dependiente resistencia a la fractura. Los resultados no fueron estadísticamente significativos cuando se evaluaron los postes metálicos a $5 \mathrm{~mm}$, que se fracturaron con cargas de 53 a $121 \mathrm{~N}$. A $10 \mathrm{~mm}$, el mismo grupo presentó una menor resistencia a la fractura, sobre $115 \mathrm{~N}$ y una incidencia mayor de fractura de la raíz considerada como desfavorable, para efectos del retratamiento. Comparativamente, los datos obtenidos registraron respuestas a la fractura similares en los dientes restaurados con fibra de carbono y fibra de vidrio. El efecto a la fractura no parece tener relación con la longitud del poste cuando es del tipo fibra (carbono o vidrio) comparado con las respuestas para el poste de acero inoxidable. En cuanto a los planos de fractura producidos, los cambios entre los diversos materiales de los postes fueron notorios, así, para los postes de acero inoxidable las fracturas sucedieron en el tercio medio con direcciones oblicuas o transversales al borde del poste, mientras que para los de fibra de carbono y vidrio estaban cerca de la región cervical. Todas las regiones de fractura se relacionaron con las zonas de mayor concentración de tensión en el interior de la raíz.

Giovani y col. en el 2009 (15), evaluaron in-vitro la resistencia a la fractura en raíces restauradas con postes de fibra de vidrio y postes colados a diferentes longitudes de preparación de conducto. Para esto utilizaron una muestra de 60 dientes sin coronas clínicas con tratamiento endodóntico y preparaciones radiculares a $6 \mathrm{~mm}$, $8 \mathrm{~mm}$ y $10 \mathrm{~mm}$, para cada restauración. Realizaron un análisis de Anova de dos vías para determinar la resistencia a la fractura, con respecto a la longitud de los postes y el tipo de material y una prueba de Tukey para las diferencias. En este estudio no se encontraron diferencias significativas en los grupos entre sí con respecto a la longitud de $6 \mathrm{~mm}, 8 \mathrm{~mm}$ y $10 \mathrm{~mm}(\mathrm{p}=8,669)$ para los postes colados; la diferencia con respecto a los demás grupos se estableció en los postes de fibra de vidrio, que resultaron bajo estas condiciones experimentales, con una mayor resistencia a la fractura dependiente de la longitud. En esta experiencia se probó que cuando se aplican fuerzas compresivas sobre la corona, se aumenta la resistencia a la fractura, cuando el poste de fibra de vidrio está cementado a una mayor longitud.

La longitud de los postes colados no afecta la resistencia radicular a la fractura y los autores consideran que estas son dadas por la rigidez de los mismos. En este estudio la localización de las fracturas fue predominante en la región más apical, con una localización desfavorable para la restauración. Bajo estas condiciones, los datos parecen favorecer la conclusión que los postes de fibra de vidrio son una alternativa viable para aumentar la resistencia a la fractura. Esto puede explicarse porque los postes de fibra de vidrio presentan un módulo de elasticidad similar a la dentina, y 
Tabla 1. Estudios incluidos en la revisión sistemática de la literatura

\begin{tabular}{|c|c|c|c|}
\hline Título del estudio & Autores y revistas & Año & Resultados \\
\hline $\begin{array}{l}\text { Fracture resistance of } \\
\text { compromised endodontically } \\
\text { treated teeth restored with } \\
\text { bonded post and cores: An in } \\
\text { vitro study. }{ }^{34}\end{array}$ & $\begin{array}{l}\text { MAROULAKOS G, NAGY W, } \\
\text { KONTOGIORGOS E. The } \\
\text { Journal of Prosthetic } \\
\text { Dentistry. (2015), 114:390- } \\
397 .\end{array}$ & 2015 & $\begin{array}{l}\text { Estadísticamente se encontró una diferencia } \\
\text { significativa en los tres grupos. Los postes } \\
\text { colados con aleaciones de oro tuvieron un mejor } \\
\text { comportamiento de resistencia a la fractura que los } \\
\text { postes de fibra de vidrio o de titanio. Los dientes } \\
\text { restaurados con postes de fibra de vidrio mostraron } \\
\text { un desalojo por falla adhesiva en la interfase entre } \\
\text { el poste y la dentina radicular. }\end{array}$ \\
\hline
\end{tabular}

Influence of post material and length on endodontically treated incisors: An in vitro and finite element study. ${ }^{33}$
CHUANG S, YAMAN P, HERRERO A, DENNISON J, CHAN C. J Prosthet Dent 2010 (2010), 104:379-388.
En una muestra de 60 incisivos se utilizaron tres tipos de postes pre-fabricados: fibra de vidrio, fibra de carbón y acero inoxidable. El incremento en la longitud de los postes no se relacionó con las fracturas producidas. El empleo de postes metálicos largos reduce la resistencia a la fractura. Los postes de fibra de vidrio proveen una resistencia mayor a fracturarse. La mayor concentración de esfuerzos analizados por metodología de elementos finitos se localizó en la región cervical para el caso de los dientes tratados con fibra de vidrio.
In vitro fracture resistance of glass-fiber and cast metal posts with different lengths. ${ }^{15}$
GIOVANI A, VANSAL L, SOUSA M, PAULINO S. J Prosthet Dent. (2009), 101:183-188.
Los postes de fibra de vidrio de $10 \mathrm{~mm}$ tuvieron la mejor respuesta a la fractura. Los postes colados tuvieron una resistencia a la fractura mayor en comparación a los postes de fibra de vidrio cuando se compara por el material. El patrón de fractura de los postes de fibra de vidrio se concentró en el área cervical y media radicular en comparación con los postes colados que se concentró en la zona apical haciendo desfavorable su retratamiento restaurador.
Microleakage and fracture patterns of teeth restored with different posts under dynamic loading. ${ }^{36}$
JUNG S, MIN K, CHANG S, PARK S, KWON S, BAE J. J Prosthed Dent. (2007), 98:270-276.
El estudio revelo que para los postes colados se presentó una microfiltración mayor en comparación con los postes de fibra de vidrio. Los autores no reportan si esté patrón está influenciado por el material de cementación. Sin embargo, sugieren que los postes cementados con resina compuesta son los que facilitan el desalojo.

La fractura de los dientes restaurados con postes colados presentó un patrón desfavorable, es decir, una fractura que no permite un retratamiento ya que sobrepasa el tercio medio radicular, a diferencia de los postes de fibra de vidrio que presentan una fractura favorable, es decir aquella que no sobrepasaba el tercio cervical radicular. 
cuando son sometidos a cargas compresivas pueden absorber mejor las fuerzas a lo largo de la raíz. Cuando las fracturas ocurren se presentan principalmente en la zona cervical radicular.

Jung SH y col. en el 2007 (36) evaluaron 40 incisivos mandibulares que fueron seccionados a nivel de la unión amelocementaria y luego tratados endodonticamente. Estos dientes fueron divididos en 4 grupos $(n=10)$ : Postes colados, postes metálicos prefabricados, postes de fibra de vidrio reforzado con resina compuesta y postes cerámicos. Después de cementar los postes se aplicó una carga intermitente de $98 \mathrm{~N}$ a $1 \mathrm{~Hz}$ durante 50.000 ciclos a un ángulo de $135^{\circ}$ del eje longitudinal de los dientes restaurados. Posteriormente, se sometieron a tinción con fucsina básica a concentración de $0.5 \%$ con el objeto de percibir los patrones de fractura; la relación de la superficie teñida con el área total de la superficie radicular se determinó con un analizador de imágenes. Los datos se analizaron con la prueba ANOVA de una vía con la variable fractura en relación con el tipo de material. Los patrones de fractura de los dientes fueron clasificados de acuerdo a sus líneas de propagación, definida como la línea de fractura expuesta en uno de los tercios de la raíz.

Se presentaron diferencias significativas entre las medias de todos los grupos $p=0,001$. El grupo de postes colados mostro significativamente un nivel más alto de microfiltración en comparación con los otros grupos. En cuanto al modo de fracaso el grupo de postes de fibra de vidrio reforzado con resina compuesta y el grupo de postes cerámicos mostraron una menor microfiltración y patrones de fractura que favorecían el retratamiento.

\section{Discusión}

Los postes de fibra de vidrio y los postes colados presentan un alto riesgo de fallas biomecánicas a mediano o largo plazo, debido a la gran pérdida de estructura dental resultado de caries preexistente y por el mismo tratamiento endodontico (37).

Varvara y col. en el 2007 (35), aunque no trabajaron con postes de fibra de vidrio, si observaron un aspecto importante con respecto al efecto de la altura de la dentina residual o remanente dentinal, que no fue valorado en ninguno de los 4 artículos tamizados en la revisión. Varvara demuestra que bajo las condiciones experimentales trazadas, al aumentar el remanente dentinal se presenta una mayor resistencia a la fractura, lo cual, favorece los retratamientos de rehabilitación. Es importante una obturación mínima de 3 a $5 \mathrm{~mm}$ de gutapercha en el ápice para mantener un adecuado selle $(\underline{38}, \underline{39})$ y la disponibilidad de $2 \mathrm{~mm}$ de estructura remanente coronal a la línea terminal de la preparación que mejore la resistencia a la fractura y que de esta manera provea retención a la corona, lo que genera un efecto de abrarazadera que previene la fractura radicular del poste y un desalojamiento del mismo (느).

En el estudio in vitro de Giovani y col. en 2009 (15) se demostró que la longitud de los postes no tiene relación con la resistencia a la fractura para las condiciones experimentales probadas, con cargas sobre postes colados y postes de fibra de vidrio; situación que fue refrendada por Chuang y col. en el 2010 (34) con postes de acero inoxidable, fibra de carbono y fibra de vidrio.

Otro hallazgo importante, en la presente revisión sucede cuando los diferentes autores, comparan el material del poste con respecto a la resistencia a la fractura. Demostraron para las condiciones experimentales, que existen diferencias entre los 
materiales y la respuesta a las fuerzas aplicadas en cuanto a la presencia de fracturas favorables o no para los retratamientos de rehabilitación. En todo caso, estas respuestas parecen favorecer especialmente a los postes de fibra de vidrio en los que las fracturas, cuando suceden, se presentan sobre el tercio cervical radicular lo que es considerado como favorable. En algunos casos la respuesta de los postes de fibra de vidrio están relacionadas directamente con el desalojo del poste sin daño a la estructura radicular $(15, \underline{33}, \underline{34})$. Cuando esto ocurre se observa que al preservar la estructura dental se reduce la posibilidad de fracturas radiculares $(\underline{41}, 42)$. Esta respuesta encontrada parece estar apoyada, también, por otros estudios como los de Dilmener y col. en el 2007 (43) en donde experimentalmente hallaron que los postes no metálicos tienen mayor resistencia a la fractura, de manera importante señalan los autores, que esta respuesta se debe al módulo de elasticidad de los materiales utilizados que suelen ser similares a los de las dentina.

Otra limitación en los estudios evaluados se presenta sobre el hecho de que no fueron estudiados en ninguno de los casos, el compromiso que tiene sobre la respuesta a la fractura, el diámetro de los postes. Stern y Hirshfeld (44) sugieren que el ancho del poste no debe ser mayor a un tercio del ancho radicular con el objetivo de no debilitar el remanente radicular. Los dientes endodónticamente tratados y rehabilitados con postes son más propensos a las fracturas que aquellos dientes rehabilitados sin tratamiento de conducto previo (ㄴ5-47).

Jung y col. en el 2007 (36) realizaron aportes importantes en el estudio de las formas y las posiciones de las fracturas en la estructura radicular. Ellos observaron la microfiltración y los patrones de fractura de dientes incisivos inferiores restaurados con diversos materiales, encontrando que en el tipo de fractura para los postes con fibra de vidrio, las líneas se limitaban al tercio cervical radicular, mientras que las líneas de fractura que sobrepasaban el tercio medio radicular se encontraron en los grupos de postes metálicos o colados. Más adelante en 2012 Chang y col. (30), hallaron que la microfiltración marginal precede a la pérdida de retención del poste, situación que es probable que ocasione una fractura radicular o fractura del poste y puede ser el precursor de un fracaso en un diente rehabilitado con retenedor intraradicular $(\underline{48}, \underline{49})$. Concluyen los autores en este estudio, que los dientes tratados con postes colados tienen un nivel mayor de microfiltración comparado con los postes de fibra de vidrio (37).

Las limitaciones de este estudio se encuentran relacionadas con los escasos artículos que cumplieron con criterios de elegibilidad robustos para sacar conclusiones. Adicionalmente, la variabilidad debida a condiciones como marcas comerciales, estructuras de dientes anteriores con formas y conductos radiculares no estandarizados y ajustes experimentales diversos, en los que se utilizaron condiciones cambiantes en cuanto a la aplicación de fuerzas y muñones restaurados con materiales disímiles entre otros. En este sentido, Monticelli y colaboradores en 2008, sugerieron la necesidad de ampliar la investigación para lograr evidencia sólida en cuanto a la indicación correcta para el uso de postes y el conocimiento a fondo de las variables que inciden en su precisa aplicación clínica (으).

\section{Conclusiones}

Los resultados consignados en los anteriores artículos revisados sugieren que la longitud del poste no tiene que ver con la resistencia a la fractura de manera significativa, adicionalmente, que la presencia de microfiltración es un factor de riesgo para fractura de la raíz, del poste o desalojo del mismo. 
En cuanto al material del que está fabricado el poste, los autores seleccionados afirman que es un factor a considerar en la respuesta a las fuerzas compresivas y tensionales que se suceden sobre la raíz y que además están relacionados de manera importante con las fracturas favorables o no para el retratamiento por rehabilitación.

Aunque existen diversas condiciones experimentales, los resultados consensuados indican que los postes de fibra de vidrio están entre las mejores alternativas cuando se presentan fuerzas tensionales importantes sobre dientes anteriores y son una opción un poco más favorable que los de metal o colados.

Según las condiciones revisadas en los artículos se debería ampliar la investigación sobre esta temáticas haciendo énfasis en el estudio de las influencias sobre la respuesta a las fuerzas aplicadas dependientes del diámetro de los postes y adicionalmente estudiar de manera separada el efecto del material de cementación. Otra situación que puede ser estudiada es el efecto que tiene bajo estas mismas condiciones experimentales el cementar postes con diversas alturas del remanente dentinal.

\section{Bibliografía}

1. Balbosh Am Kern M. Effect of surface treatment on retention of glass fiber endodontic post. Journal of Prosthetic Dentistry. 2006; 95(3):218-223. link

2. Baraban D. The restoration of endodontically treated teeth: an update. J Prosthed Dent. 1988; 59(5):553-558. link

3. Schiavetti R, García F, Toledano M, Mazzitelli C, Barlattani A, Ferrari M, Comparison of fracture resistance of bonded glass fiber posts at different lengths. Am J Dent. 2010; 23(4):227-230. link

4. Plotino G, Grande N, Bedini R, Pameijer C, Somma F. Flexural properties of endodontic posts and human root dentin. Dental Materials.2007. 23(9):1129-1135. link

5. Morgano S, Brackett S. Foundation restorations in fixed prosthodontics: current knowledge and future needs- J Prosthet Dent. 1999; 82(6):643-657. link

6. Schmitter M, Hamadi K, Rammelsberg P- Survival of two post systems-fiveyear results of a randomized clinical trial. Quintessence International. 2011; 42(10):843-850. link

7. Goracci C, Ferrari M. Current perspectives on post systems: a literature review. Australian Dental Journal. 2011; 56(Suppl1):77-83. link

8. Goodacre C, Spolnil K. The prosthodontic management of endodontically treated teeth: a literature review. Part I. Success and failure data, treatment concepts. J Proshtodont. 1994; 3(4):243-250. link

9. Breschi L, Mazzoni A, Ferrari M. Adhesion to intra radicular dentin en: Ferrari M. Fiber posts and endodontically treated teeth: a compendium of scientific and clinical perspectives. Wendywood: Moderns Dentistry Media, 2008(1):15-37. 
10. Mamootil K, Messer $\mathrm{H}$. Penetration of dentinal tubules by endodontic sealer cements in extracted teeth and in vivo. Int Endod J. 2007; 40(11):873-881. link

11. Saupe W, Glusking A, Radke R. A comparative study of fracture resistance between morphologic dowel and cores and a resin reinforced dowel system in the intraradicular restoration of structurally compromised roots. Quintessence Int. 1996; 27(7):483-491. link

12. Stern N, Hirshfeld Z. Principles of preparing endodontically treated teeth for dowel and core restorations. J Prosthet dent. 1973(2); 30:162-165. link

13. Vichi A, Ferrari M, Davidson C. Influence of ceramic and cement thickness on the masking of various types of opaque posts. J Prosthet Dent. 2000; 83(4):412-417. link

14. Toksavul S, Toman M, Uylgan B, Schmage P, Nergiz I. Effect of luting agents and reconstruction techniques on the fracture resistance of pre-fabricated post systems. Journal of Oral Rehabilitation.2005; 32(6):433-440. link

15. Giovani A, Vansan L, de Sousaneto M, Paulino S. In vitro fracture resistance of glass-fiber and cast metal posts with different lengths. J Prosthet Dent. 2009; 101(3):183-188. link

16. Parisi C, Valandro L, Ciocca L, Gatto M, Baldissara P. Clinical outcomes and success rates of quartz fiber post restorations: A retrospective study. The Journal of Prosthetic dentistry. 2015; 114(3): 367-372. link

17. Fernandes A, Dessai G. Factors affecting the fracture resistance of post-core reconstructed teeth: a review. Int J Prosthodont. 2001; 14(4):355-363. Link

18. Schwartz R. Fundamentals of operative dentistry.1 ed. Londres. Quintessense Publishing. 1996:. p325. link

19. Nasr H, Eskander M, Zaki A. The luting efficacy of cement in bonding dowels of different metal. Egypt dent J. 1987; 33(2):155-171. link

20. Tjan A, Tjan A, Greive J. Effects of various cementation methods on the retention of prefabricated post. J Prosthet Dent. 1987; 58(3):309-311. link

21. Hochman N, Feinzaig I, Zalkind M. Effect of design of pre-fabricated post and post heads on the retention of various cements and core materials. J Oral Rehabil. 2003; 30(7): 702-707. link

22. Zhou L. Wang Q. Comparison of fracture resistance between cast posts and fiber posts: a metanalysis of literature. J Endod. 2013. 39(1):11-15. link

23. Polly M, Nicholls J, Junge T, Phillips K. Load fatigue of teeth with different ferrule lengths, restored with fiber posts, composite resin cores, and all ceramic crowns. J Prosthet Dent. 2009; 102(4):229-234. link

24. Berastain Jm Domínguez E y Pagan L. Diagnóstico y tratamiento de la oclusión. En: Odontología Integral Actualizada. Brasil: 2006. Editorial Artes Médicas Ltda, p64-65. 
25. Verissimo C, Simamoto P, Soares C, Noritomi P, Freita P. Effect of the crown post and remaining coronal dentin on the biomechanical behavior of endodontically treated maxillary central incisors. J Prosthet Dent 2014; 111(3):234-246. link

26. Chan R. Restoration of endodontically treated teeth. Aust Prosthodont J. 1988; 2(1):55-66.

27. Fernandes A, Shetty S, Coutihho. Factors determining post selection; a literature review- J Prosthet Dent. 2003; 90(6):556-562. link

28. Dikbas I, Tanalp J. An overview of clinical studies on fiber post systems. The Scientific world Journal. 2013; Article ID 171380. doi:10.1155/2013/171380.

29. Vire D. Failure of endodontically treated teeth: Classification and evaluation. J Endod. 1991; 17(7):338-342. link

30. Chang J, Soo I, Cheung G. Evaluation of fiber post-supported restorations under simulated occlusal loading. J Prosthet Dent. 2012; 108(3):158-164. link

31. Al-Harbi F, Nathanson D. In vitro assesment of retention of four esthetic dowels to resin core foundation and teeth J Prosthet Dent. 2003; 90(6):547-555. link

32. Pedro.org.au [Internet].Australia; Australian Physiotherapy Asociation; 2016 [actualizado 2 de mayo de 2016; citado 8 feb 2016]. Disponible en: http://www.pedro.org.au/wp-content/uploads/PEDro scale spanish.pdf.

33. Chuang S, Yaman P, Herrero A, Dennison J, Chan C. Influence of post material and length on endodontically treated incisors: An in vitro and finite element study. J Prosthet Dent. 2010; 104(6):379-388. link

34. Maroulakos G, Nagy W, Kontogiorgos E. Fracture resistance of compromised endodontically treated teeth restored with bonded post and cores: An in vitro study. The Journal of Prosthetic Dentistry. 2015; 114(3):390-397. Link

35. Varvara G, Perinetti G, Dilorio D, Murmura G, Caputi S. In vitro evaluation of fracture resistance and failure mode of internally restored endodontically treated maxillary incisors with differing heights of residual dentin. J Prosthet Dent. 2007; 98(5):365-372. link

36. Jung S, Min K, Chang S, Park S, Kwon S, Bae J. Microleakage and fracture patterns of teeth restored with different posts under dynamic loading. J Prosthet Dent. 2007; 98(4):270-276. link

37. Hyedecke G, Peters M. The restoration of endodontically treated, single rooted teeth with cast or direct posts and cores: A systematic review. J Prosthet Dent. 2002; 87(2):380-386. link

38. Mattison G, Delivanis P, Thacker R, Hassel K. Effect of post preparation on the apical seal. J Prosthet Dent. 1984; 51(6):785-589. link

39. Pereira J, Ornelas F, Rodríguez P, Lins A. Effect of a crown ferrule on the fracture resistance of endodontically treated teeth restored with prefabricated posts. The journal of prosthetic dentistry. 2006; 95(1):50-54. link 
40. Gegauff A. Effect of crown lengthering and ferrule placement on static load failure of cementes cast post cores and crowns. J Prosthet Dent. 2000; 84(2):169179. link

41. Trabert K, Caputo A. Abou Rass M. Tooth fracture a comparison of endodontic and restorative treatments. J endod. 1978; 4(11):341-345. link

42. Maceri F, Martignoni M, Vairo G Mechanical behaviour of endodontic restorations with multiple prefabricated posts: A finite element approach. . Journal of Biomechanics. 2007; 40(11):2386-2398. link

43. Dilmener F, Sipahi C, Dalkiz M. Resistance of three new esthetic post and core systems to compressive loading. J Prosthet Dent. 2006; 95(2):130-136. link

44. Stern N, Hirshfeld Z. Principles of preparing endodontically treated teeth for dowel and core restorations. J Prosthet dent. 1973; 30(2):162-165. link

45. Monga P, Sharma V, Kunar S. Comparison of fracture resistance of endodontically treated teeth using different coronal restorative materials: an in vitro study. Journal of Conservative Dentistry. 2009; 12(4):154-159. link

46. Belli S, Erdemir A, Yildirim C. Reinforcement effect of polyethylene fibre in rootfilled teeth: comparison of two restoration techniques. International Endodontic Journal. 2006; 39(2):136-142. link

47. Dikbas I, Tanalp J, Ozel E, Koksal T. Ersot M. Evaluation of the effect of different ferrule designs on the fracture resistance of endodontically treated maxillary central incisor incorporating fiber posts, composite cores and cros restorations. Journal of Contemporary Dental Practice. 2007; 8(7):62-69. link

48. Vire D. Failure of endodontically treated teeth: Classification and evaluation. J Endod. 1991; 17(7):338-342. link

49. Amarnath G, Swetha M, MuddugangadharB, Sonika R, Garg A, Rao T. Effect of post material and length on fracture resistance of endodontically treated premolar: A in vitro study. Journal of international Oral health. 2015; 7(7):22-28. link

50. Monticelli F, Osorio R, Sadek F, Radovic I, Toledano M, Ferrari M. Surface treatments for improving bond strength to prefabricated fiber posts: A literature Review. Operative Dentistry. 2008; 33(3):346-355. link 